\title{
The requirement for Phr1 in CNS axon tract formation reveals the corticostriatal boundary as a choice point for cortical axons
}

\author{
A. Joseph Bloom, ${ }^{1}$ Bradley R. Miller, ${ }^{1}$ Joshua R. Sanes, ${ }^{2}$ and Aaron DiAntonio ${ }^{1,3}$ \\ ${ }^{1}$ Department of Molecular Biology and Pharmacology, Washington University, Saint Louis, Missouri 63110, USA; \\ ${ }^{2}$ Department of Molecular and Cellular Biology, Harvard University, Cambridge, Massachusetts 02138, USA
}

\begin{abstract}
Phr1 is the single well-conserved murine ortholog of the invertebrate ubiquitin ligase genes highwire (in Drosophila) and rpm-1 (in Caenorhabditis elegans). The function and mechanism of action of highwire and rpm-1 are similar-both cell-autonomously regulate synaptogenesis by down-regulating the ortholog of the mitogen-activated protein kinase kinase kinase dual leucine zipper kinase (MAPKKK DLK). Here, using a targeted conditional mutant, we demonstrate that Phr1 also plays essential roles in mammalian neural development. As in invertebrates, Phr1 functions cell-autonomously to sculpt motor nerve terminals. In addition, Phr1 plays essential roles in the formation of major CNS axon tracts including those of the internal capsule, in part via cell-nonautonomous mechanisms, and these results reveal a choice point for cortical axons at the corticostriatal boundary. Furthermore, whereas the neurite morphology phenotypes of highwire and rpm-1 are suppressed by loss of DLK in flies and worms, Phr1-dependent CNS defects persist in Phr1, DLK double mutants. Thus, in the mammalian nervous system Phr1 is required for formation of major CNS axon tracts via a mechanism that is both cell-nonautonomous and independent of DLK.
\end{abstract}

[Keywords: Axon guidance; synapse formation; internal capsule; neuromuscular junction; DLK]

Supplemental material is available at http://www.genesdev.org.

Received July 11, 2007; revised version accepted August 17, 2007.

Phr1 is the single murine ortholog of invertebrate ubiquitin ligases that regulate synapse growth and morphology. Loss of highwire, the Drosophila ortholog, causes dramatic synaptic overgrowth and a physiological deficit at the larval neuromuscular junction (NMJ) (Wan et al. 2000; DiAntonio et al. 2001), while mutations of the Caenorhabditis elegans ortholog rpm-1 cause defects in the morphology, spacing, and number of presynaptic active zones (Schaefer et al. 2000; Zhen et al. 2000). This synaptic function is conserved in vertebrates, as mutants of esrom, the zebrafish ortholog of Phr1, display defects in synaptic target selection during retinotectal mapping (D'Souza et al. 2005). All of these functions of the PHR family are cell-autonomous, likely reflecting actions of the protein in nerve terminals (Schaefer et al. 2000; Zhen et al. 2000; D'Souza et al. 2005; Wu et al. 2005). As befits a large, multidomain protein, PHR family members interact with many candidate targets (Guo et al. 1998; McCabe et al. 2004; Pierre et al. 2004; Murthy et al. 2004);

${ }^{3}$ Corresponding author.

E-MAIL diantonio@wustl.edu; FAX (314) 362-7058.

Article published online ahead of print. Article and publication date are online at http://www.genesdev.org/cgi/doi/10.1101/gad.1592107. however, genetic data demonstrates that one critical substrate of Highwire/RPM-1 is the mitogen-activated protein kinase kinase kinase (MAPKKK) called Wallenda in Drosophila and DLK (dual leucine zipper kinase) in C. elegans. In both worms and flies, levels of this kinase are increased in the absence of highwire/rpm-1, synaptic defects in highwire/rpm-1 mutants are suppressed by loss of wallenda/DLK, and increasing the level of Wallenda/ DLK is sufficient to phenocopy the synaptic defects of highwire/rpm-1 mutants (Nakata et al. 2005; Collins et al. 2006).

To assess the role of Phr1 in mammalian neural development, we have generated a targeted conditional Phr1 knockout allele in the mouse. Conditional excision of Phr1 in motoneurons demonstrates that, as in invertebrates, Phr1 is required cell-autonomously for normal neuromuscular synaptogenesis, consistent with previous analysis of mice with large chromosomal deletions that remove Phr1 and neighboring genes (Burgess et al. 2004). In addition, we have discovered severe and specific defects in the establishment of major axon tracts in the CNS, including a failure of corticofugal and thalamocortical axons to enter the subcortical telencephalon and form an internal capsule, the major avenue for informa- 
tion flow between the cerebral cortex and the rest of the nervous system. While all previously described functions of Phr1 orthologs are cell-autonomous, conditional deletion of $P h r 1$ demonstrates a cell-nonautonomous requirement for Phr1 in the guidance of cortical axons. These findings suggest that $P h r 1$ is required to generate a permissive environment for corticofugal and thalamocortical axons as they pass into the subcortical telencephalon, and reveals the corticostriatal boundary as a choice point for descending cortical axons. Finally, we tested the interaction of Phr1 with DLK (Holzman et al. 1994; Reddy and Pleasure 1994), the murine ortholog of the MAPKKK that is a critical substrate of the invertebrate PHR genes. We generated DLK mutants and Phr1 $D L K$ double mutants and find that the disruption of the internal capsule persists in double mutants, demonstrating that this phenotype is not due to up-regulation of DLK. Therefore, in addition to its evolutionarily conserved, cell-autonomous role in regulating synapse development, these studies have demonstrated three novel aspects of Phr1 function in the mammalian nervous system: Phr1 is (1) required for formation of major axon tracts in the CNS, and it can act via a mechanism that is both (2) cell-nonautonomous and (3) independent of $D L K$.

\section{Results}

Loss of Phr1 causes defects in neuromuscular development

Phr1 is expressed widely in the developing and adult mouse CNS (Yang et al. 2002; Burgess et al. 2004; Santos et al. 2006). To determine the function of Phr1, we generated conditional Phr1 knockout mice utilizing the Crelox system (Fig. 1A). The Phr1 gene contains $>80$ exons spanning $>200 \mathrm{~kb}$. The floxed exons encode 70 amino acids adjacent to the RCC1 domain, a highly conserved domain necessary for the function of highwire in Drosophila (C. $\mathrm{Wu}$, pers. comm.). A constitutive knockout allele was subsequently derived from the floxed allele and mutations were confirmed by Southern blot (Fig. 1B), genomic PCR (data not shown), and RT-PCR (see below). Phr1 constitutive knockout mice die at birth without taking a breath, their phrenic nerves are markedly narrower and contain fewer axons than controls (Fig. 1C-E), and they often lack innervation of the most ventral region of the diaphragm (Supplementary Fig. S1). Other peripheral nerves are properly formed and located, albeit thin. Motor axons terminate in normal-appearing presynaptic NMJ structures apposed to endplates, but they also extend dysmorphic extrasynaptic varicosities filled with axonal and synaptic markers (Fig. 1F,G). These data confirm that Phr1 is responsible for the neuromuscular phenotypes reported by Burgess et al. (2004) in chromosomal deficiency mice lacking Phr1 and two adjacent genes.

Since there are fewer axons in the phrenic nerve, one might expect to find fewer post-synaptic endplates on the muscle. However, innervated portions of the diaphragm in Phr1 mutants have similar numbers of endplates, as in control animals, although the endplates are more densely packed within a narrower endplate band (Fig. 1H-K). All endplates are apposed to presynaptic nerve terminals and the location of the endplate band is not otherwise altered. The defects at the NMJ are summarized in Supplementary Figure S2. An implication of these results is that the average motor unit size (the complement of endplates innervated by a single neuron) is increased in Phr1 mutants; i.e., each motoneuron in the knockout innervates more endplates than in controls. This is analogous to the NMJ defect in Drosophila mutants for highwire, in which each motor axon develops a greater than usual number of synaptic boutons.

Presynaptic phenotypes can result from defects in either motoneurons or muscle (Sanes and Lichtman 1999), so it was important to determine whether Phr1 defects at the NMJ are due to loss of Phr1 in motoneurons. To this end we mated Phr1-floxed allele mice to the motoneuron-specific Cre driver HB9-Cre. Because HB9-Cre mice express Cre selectively in motoneurons (Fox et al. 2007), the resulting offspring develop NMJs that lack Phr1 only in the presynaptic cell. The viability of motoneuron-specific mutants is variable, unlike that of constitutive Phr1 knockout: Some animals are stillborn, but many survive to adulthood and are fertile. Thus, defects in the neuromuscular system contribute importantly to the lethality in the constitutive Phr1 knockout, although Phr1 function in other tissues may also contribute.

The morphological defect at the NMJ in conditional motoneuron Phr1 knockouts is similar to that of constitutive knockouts-numerous neurofilament-rich sprouts extend beyond normally innervated endplates (Fig. 1L,M). Conditional Phr1 knockouts that display this altered NMJ morphology can have fully innervated diaphragms, indicating that the cell morphology defect apparent across the diaphragm is not secondary to the lack of innervation of the ventral region of the diaphragm. Therefore, mouse Phr1, like its Drosophila and C. elegans orthologs (Schaefer et al. 2000; Zhen et al. 2000; Wu et al. 2005), functions cell-autonomously in motoneurons to regulate synaptic development.

\section{Phr1 mutants lack retinal innervation of the thalamus}

The thinner peripheral nerves and partially uninnervated diaphragm in Phr1 knockout mice lead us to wonder whether Phr1 functions in axon outgrowth or guidance as well as synaptic development. To investigate guidance in an experimentally accessible nerve, we analyzed the projection of retinal ganglion cells, which express Phr1 (data not shown), to the lateral geniculate nucleus (LGN) of the thalamus. Labeling the retinal projections with the lipophylic dye DiI, we found no innervation of the LGN in neonatal Phr1 constitutive mutants $(n=4)$, whereas control littermates showed robust labeling of the optic tracts and dorsal thalamus $(n=2)$ (Fig. 2). In knockouts, the extent of the defect varied, but axons 


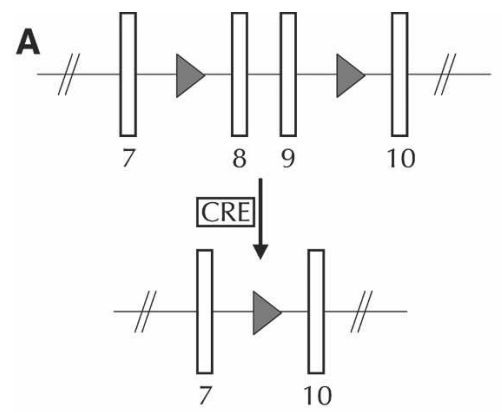

B

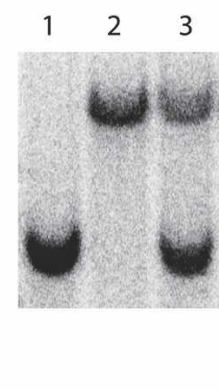

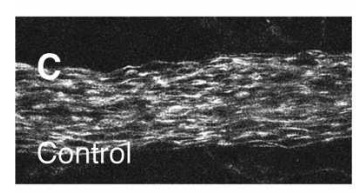
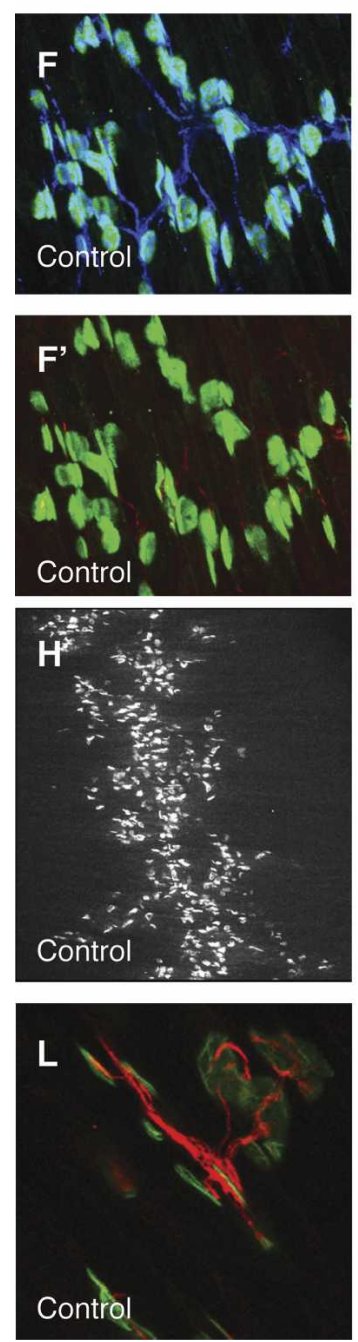
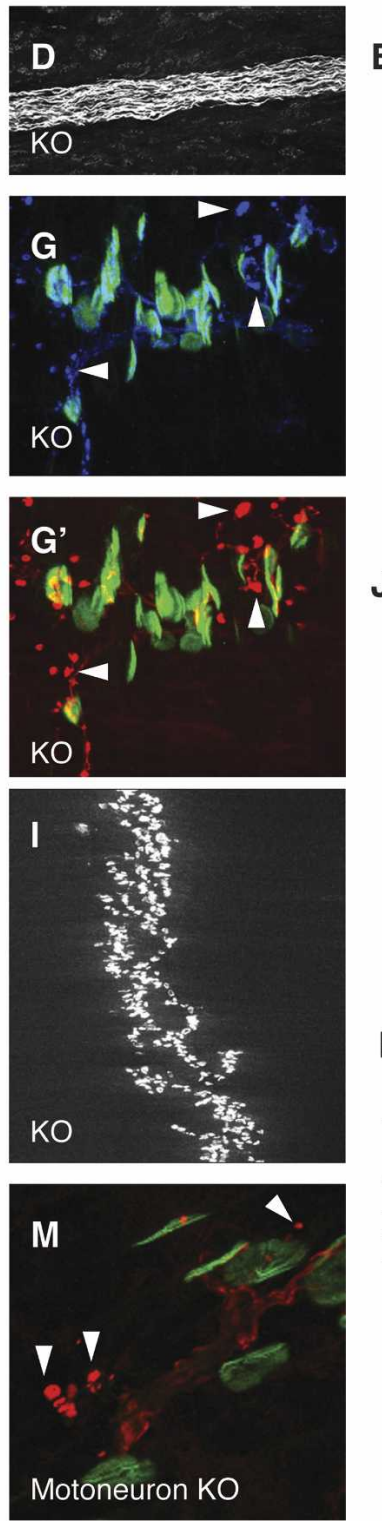

$E$
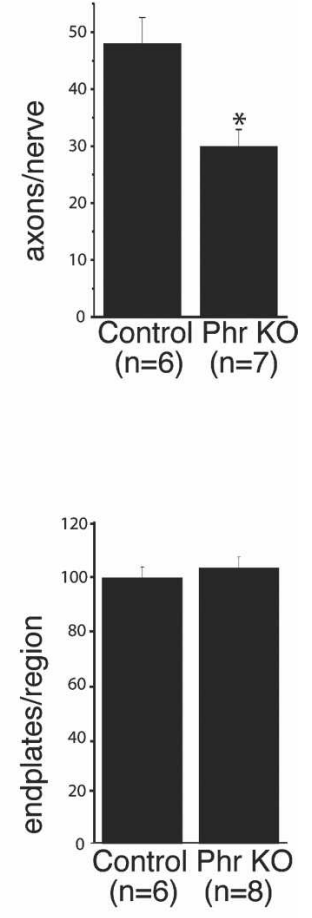

K

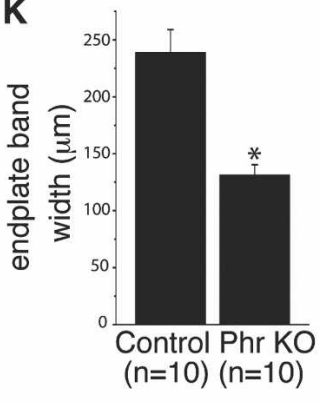

Figure 1. Phr1 functions in the motoneuron to restrain presynaptic sprouting. $(A)$ Schematic of the targeted Phr1 mutant allele. Two LoxP sites (triangles) were introduced by homologous recombination into the Phr1 locus flanking the eighth and ninth translated exons to create the "floxed" allele. In the presence of Cre, the two LoxP sites recombine and excise the intervening genomic DNA to become the constitutive knockout allele. (B) Southern analysis demonstrates the presence of the targeted chromosome in the Phr1 knockout homozygote (lane 1), the wild-type chromosome in a wild-type littermate (lane 2), and both chromosomes in a heterozygote (lane 3). Phrenic nerves stained with neurofilament are shown at E18.5 from control $(C)$ and Phr1 constitutive knockout (KO) (D) embryos. Quantification of the mean number of axons in the phrenic nerve is shown in $E$. NMJs from E18.5 control $\left(F, F^{\prime}\right)$ and $P h r 1$ constitutive knockout $\left(G, G^{\prime}\right)$ diaphragms are shown stained for acetylcholine receptors with bungarotoxin (green), synaptophysin (blue), and neurofilament (red). In the Phr1 constitutive knockout, examples of extrasynaptic sprouts containing neurofilament and synaptophysin are highlighted with arrowheads. The endplate band at E18.5 stained for acetylcholine receptors with bungarotoxin is shown for control $(H)$ and Phr1 constitutive knockout $(I)$ diaphragms. Quantification of the mean number of endplates within a defined muscle region as a percentage of wild type is shown in $J$. Quantification of the mean endplate band width is shown in $K$. NMJs from P3 control (L) (Hb9-Cre; +) and Phr1 motoneuron knockout (M) (Hb9-Cre; Phr1 ${ }^{\text {flox }} / \mathrm{Phr}^{\text {flox }}$ ) diaphragms are shown stained for acetylcholine receptors with bungarotoxin (green) and neurofilament (red). In the Phr1 motoneuron knockout, examples of extrasynaptic sprouts containing neurofilament are highlighted with arrowheads. $\left(^{\star}\right) P<0.01$. Data are presented as mean \pm SEM.

always left the retina and formed an optic nerve and often reached and crossed the optic chiasm; in one animal, a few labeled axons were detected at the beginning of the optic tract, but none reached the dorsal thalamus. Hence, Phr1 is required for retinal innervation of the brain.

\section{Mice lacking Phrl display CNS morphology defects}

The broad expression of Phr1 throughout the CNS, along with the guidance defects of retinal projections, lead us to extend our investigations to major axon tracts within the brain (Fig. 3). Several gross morphological defects are 

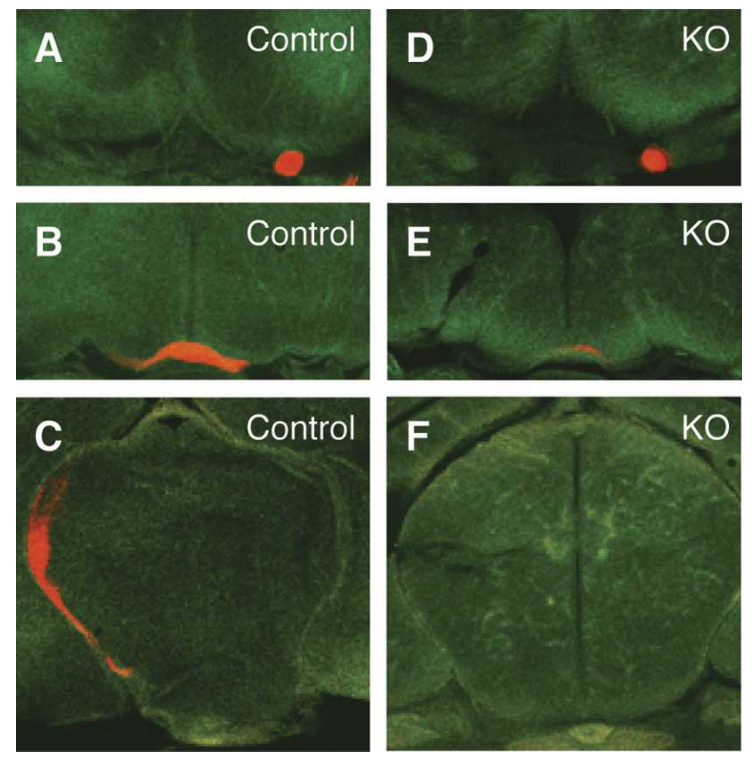

Figure 2. Phr1 mutants lack retinal innervation of the thalamus. Green Nissl-stained serial coronal sections from P0 heads labeled with DiI (red) in the retina. Labeled optic nerves $(A, D)$, optic chiasm $(B, E)$, and thalamus $(C, F)$ from control and Phr1 constitutive knockouts. Axon labeling is greatly reduced at the knockout chiasm and is absent in the knockout thalamus.

apparent in the Phr1 knockout brain: The volume of the lateral ventricles is greatly increased, extending into the olfactory bulb in mutants, and hippocampal formations are smaller and dysmorphic (Fig. 3A-G). In the diencephalon, the anterior thalamus is reduced in size and altered in organization (Fig. 3A-G). Despite these gross changes, there are no obvious defects in the overall organization of the cerebral (Fig. 3H,I) and cerebellar cortices (Supplementary Fig. S3) at embryonic day 18.5 (E18.5) - the cerebral cortical thickness of the knockout is normal $(93 \pm 3 \%$ of control) and the thickness of the cortical plate as a percentage of the total cortical thickness is normal (Control $59.2 \pm 0.8 \%$; Phr1 knockout $62.7 \pm 0.5 \%)$, as is expression of the transcription factor Tbr1, a marker of post-mitotic neurons in the cortical plate (Fig. 3J,K; Englund et al. 2005). In addition, cell death in the CNS is not increased in Phr1 mutants at any investigated time point (E16.5 and E18.5), nor is there a significant change in cell proliferation as assessed by BrdU labeling of cells born at E12.5 and E15.5 (data not shown).

In the Phr1 mutant, striking defects are apparent in major axon tracts: The anterior commissure is absent (Supplementary Fig. S3), the corpus callosum is narrower in both the anterior-posterior and dorsal-ventral axes (see below), and there is extensive loss of neurites in the cerebral cortex (Fig. 3A-G). Additionally, Nissl staining of Phr1 mutant embryonic brains and immunohistochemistry for axon markers including L1 (Fig. 3A-G) and calretinin demonstrate the loss of corticofugal and thalamocortical projections in the subcortical telencephalon, where the internal capsule is normally located. The ha- benulopenduncular tract is short and the adjacent interpeduncular fossa exaggerated (Supplementary Fig. S3). Immunohistochemistry demonstrates a general reduction of neurofilament in brain structures, including the hippocampus, cerebral cortex, subcortical telencephalon, and thalamus (Supplementary Fig. S3). Axonal defects are not confined to long axons such as those passing through the commissures and internal capsule: There is a marked reduction in staining for neurites of GABAergic interneurons without an appreciable reduction in the number of GABAergic cell bodies in the cerebral cortex (Fig. 3L,M).

All of the above gross morphology defects are also observed in deficiency mice lacking the entire Phr1 locus as well as mice transheterozygous for the constitutive Phr1 allele and a deficiency chromosome (Fig. 3C,D,G; Supplementary Fig. S3K). Heterozygotes for the targeted Phr1 knockout allele do not display appreciable defects. The nature and severity of CNS and PNS phenotypes are indistinguishable among all three genotypes mutant for Phr1, demonstrating that our constitutive targeted allele behaves as a genetic null allele.

\section{Corpus callosal defects in Phrl mutants are region specific}

For detailed analysis of Phr1 function, we selectively deleted Phr1 from the cerebral cortex using Emx1-Cre mice. Emx1-Cre is expressed in progenitors of excitatory neurons in the cerebral cortex and hippocampal formation, including all projection neurons that contribute to the corpus callosum and internal capsule. GABAergic interneurons do not express Cre, having arisen elsewhere and subsequently migrated into the cortex. Cre expression is observed in only rare cells of subpallial structures (Gorski et al. 2002; Bareyre et al. 2005). Mice homozygous for the Phr1 floxed allele and expressing Emx1-Cre survive to become fertile adults, indicating that the lethality of Phr1 constitutive knockouts is not due to loss of Phr1 in the cortex. Emx1-Cre; Phr1 mice do, however, display many of the telencephalic defects observed in the null. These include enlarged lateral ventricles, dysmorphic hippocampal formations, and narrower corpora callossa. The callosal defect is qualitatively and quantitatively indistinguishable from that of the constitutive Phr1 knockout (Fig. 4A-C). These phenotypes persist into adulthood (data not shown).

The reduction of the corpus callosum could result from an absence of callosally directed axons or from a failure of such axons to cross the midline. In either case, the reduction might be a cortex-wide defect or restricted to specific cortical areas. To distinguish among these alternatives we performed axon-tracing experiments on E18.5 embryos using the lipophilic dye DiI. DiI placed approximately in the region of the incipient somatosensory cortex usually crossed the corpus callosum of control, cerebral cortex-specific, and constitutive mutant embryos (five out of five controls, four out of four cerebral cortex-specific knockouts, and two out of three constitutive knockouts) (Fig. 4D-F). No consistent differ- 

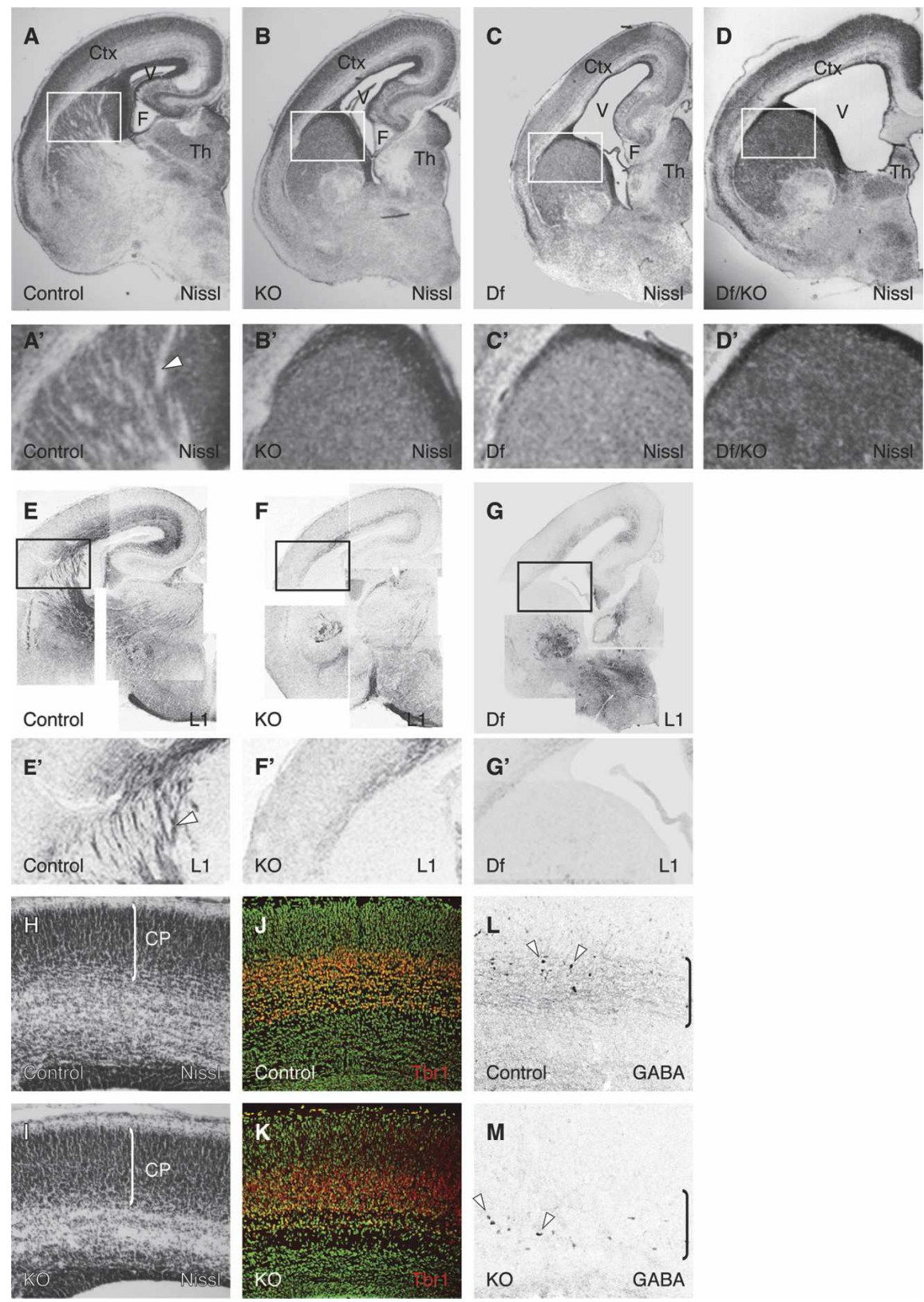

Figure 3. Phr1 constitutive knockout displays severe defects of gross morphology and axon fiber tracts in the CNS. Nissl-stained coronal sections from one side of E18.5 brains of control $\left(A, A^{\prime}\right)$, Phr1 constitutive knockout (KO) $\left(B, B^{\prime}\right)$, Phr1 deficiency mutants $(15 \mathrm{DttMb} / 15 \mathrm{DttMb})\left(C, C^{\prime}\right)$, and transheterozygotes for the constitutive allele and deficiency allele $\left(D, D^{\prime}\right)$. Nissl stains cell bodies and shows white matter tracts in relief. In the Phr1 knockout and Phr1 deficiency mutant (Df), lateral ventricles are increased in size, the hippocampus is reduced, and axon tracts running through the internal capsule are absent. Cortex (Ctx), lateral ventricle (V), fimbria $(\mathrm{F})$, and thalamus $(\mathrm{Th})$ are labeled. The boxed region is shown in $A^{\prime}, B^{\prime}, C^{\prime}$, and $D^{\prime}$ and demonstrates the presence of white matter tracts (arrowhead) in the internal capsule from the control $\left(A^{\prime}\right)$ but not from the Phr1 knockout and Phr1 deficiency mutant $\left(B^{\prime}, C^{\prime}, D^{\prime}\right)$. Staining for L1, a marker of major fiber tracts including the internal capsule, is shown in coronal sections from one side of E18.5 brains of control $\left(E, E^{\prime}\right)$, Phr1 constitutive knockout (KO) $\left(F, F^{\prime}\right)$, and $P h r 1$ deficiency mutants (Df) $\left(G, G^{\prime}\right)$. The boxed regions shown in $E^{\prime}, F^{\prime}$, and $G^{\prime}$ demonstrate the presence of L1-positive axon tracts (arrowhead) in the internal capsule from the control (E') but not of the Phr1 knockout $\left(F^{\prime}\right)$ or Phr1 deficiency mutant $\left(G^{\prime}\right)$. Coronal sections of Nissl-stained control $(H)$ and Phr1 constitutive knockout $(\mathrm{KO})(I)$ E18.5 cerebral cortex highlight the cortical plate $(\mathrm{CP})$ whose thickness (indicated by the bracket) as a percentage of the total cortical thickness is unchanged in the mutant. Coronal sections of Nissl-stained (green) and Tbrl-stained (red) control (J) and Phr1 constitutive knockout $(\mathrm{KO})(K)$ E18.5 cerebral cortex highlight that $\mathrm{Tbr}^{+}$neurons have migrated into the cortex and differentiated in similar positions in mutant and controls. Coronal sections of GABA-stained control $(L)$ and Phr1 constitutive knockout (KO) (M) E18.5 cerebral cortex show GABAergic cell bodies (arrowheads) in both the control and mutant, while highlighting the more prominent GABAergic neurite staining (bracketed regions) in the control. 


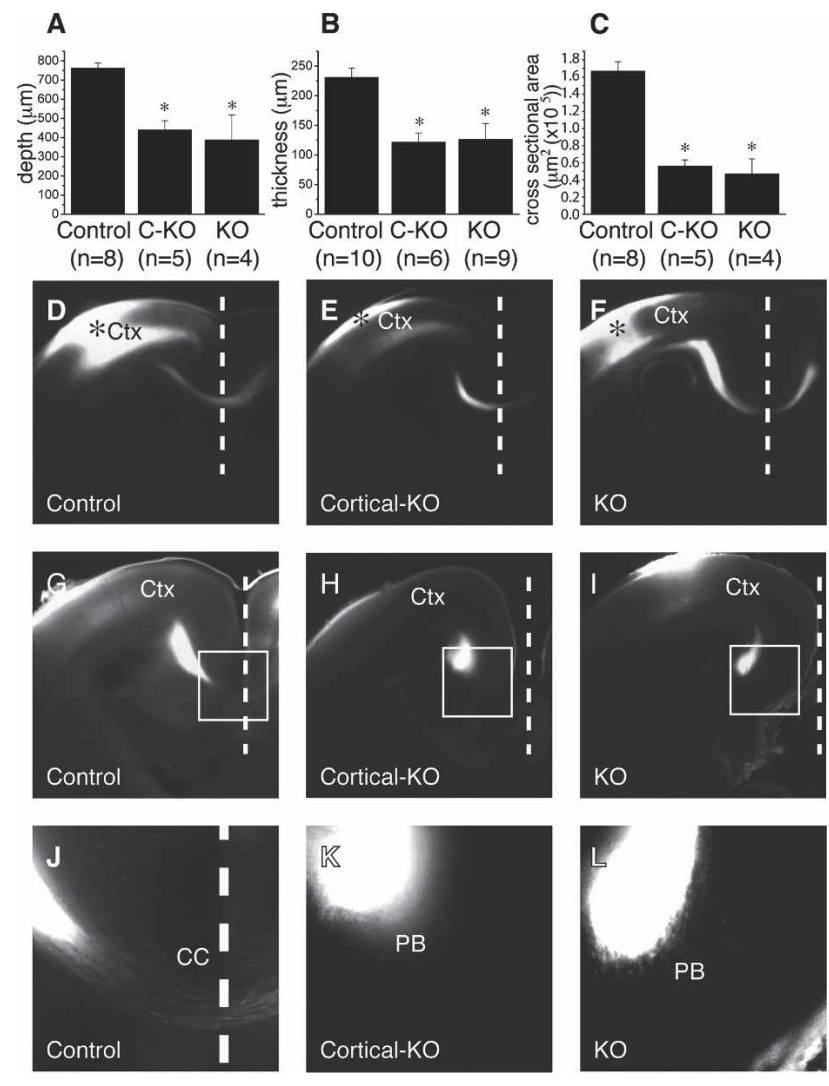

Figure 4. Corpus callosum defects in the Phr1 mutant are region specific. Quantification of corpus callosum size as measured from brain sections of E18.5 control, cerebral cortex-specific Phr1 knockout (C-KO, Emx1-Cre; Phr $\left.1^{\text {flox }} / P h r 1^{\text {flox }}\right)$, and constitutive Phr1 knockout (KO) shows the anterior-posterior depth $(A)$, dorsal-ventral thickness $(B)$, and cross-sectional area $(C)$. In $A$, the asterisk indicates $P<0.01$, in $B$, the asterisk indicates $P<0.005$, and in $C$, the asterisk indicates $P<0.0005$ compared with control, demonstrating that $P h r 1$ is required for normal corpus callosum formation. The size of the corpus callosum is not different between the cerebral cortex-specific and constitutive Phr1 alleles, demonstrating that Emx1-Cre effectively deletes Phr1 in the projection neurons contributing to the corpus callosum. DiI labeling of cortical axons is shown in E18.5 coronal sections in $D-L$. DiI placed in the somatosensory cortex (Ctx) of control $(D)$, cerebral cortex-specific Phr1 knockouts (Cortical-KO) (E), and constitutive Phr1 knockouts (KO) (F) labels axons that cross the midline (dashed line) via the corpus callosum. Asterisk indicates dye placement in the cortex. DiI placed in the visual cortex of control $(G, J)$, cerebral cortex-specific Phr1 knockouts (Cortical-KO) $(H, K)$, and constitutive Phr1 knockouts (KO) $(I, L)$ labels axons that cross the midline (dashed line) in the control, but not in the cerebral cortexspecific or constitutive $P h r 1$ knockout. The boxed region in $G-I$ is magnified in $J-L$ and highlights that axons from the visual cortex cross the midline in the control, but fail to cross and instead accumulate in "Probst bundles" in both the cerebral cortex-specific and constitutive Phr1 knockout. Data are presented as mean \pm SEM.

ence in the dye crossing was noted between controls and mutants. When DiI was placed approximately in the incipient visual cortex, it invariably crossed the corpus cal- losum of control embryos $(n=5)$ (Fig. 4G,J), although many fewer callosal axons were observed crossing from the visual cortex than from the somatosensory cortex in control animals (Fig. 4, cf. D and G). In contrast, callosal axons from the incipient visual cortex never crossed in Phr1 knockout embryos $(n=3)$ (Fig. 4I,L) or cerebral cortex-specific Phr1 knockouts $(n=4)$ (Fig. $4 \mathrm{H}, \mathrm{K})$. Rather, in Phr1 mutants, dye-labeled axons originating from the incipient visual cortex extend anteriorly and medially away from the site of dye placement and approach the midline as usual, but terminate in large bulbs known as "Probst bundles" instead of entering the corpus callosum. DiI experiments demonstrate that this defect persists until at least postnatal day 8 (P8) in cortical knockouts (data not shown), making it unlikely that the defect is due to a delay of axon outgrowth or crossing. From these results we conclude that a reduction of the corpus callosum in Phr1 mutant animals is due to a lack of crossing by axons arising from the posterior cerebral cortex, rather than a general defect throughout the cortex.

\section{Guidance defects of descending cortical and ascending thalamic projections in Phrl mutants}

Dye-tracing experiments were performed to analyze the extent of axon loss through the internal capsule of E18.5 Phr1 mutant brains. DiI-labeled axons passing through the internal capsule were apparent in all control E18.5 brains that had DiI introduced in the incipient somatosensory (Fig. 5A) or visual (data not shown) cortices ( $n=10$ experiments). Retrogradely labeled thalamic neurons were also evident in control animals (Fig. 5D). However, in identically treated E18.5 Phr1 constitutive mutant embryos, neither DiI-labeled tracks running through the subcortical telencephalon, nor retrogradely labeled cell bodies outside the cerebral cortex, were ever observed (Fig. 5C,F) ( $n=6$ experiments). Furthermore, DiI placed in the subcortical telencephalon of knockout brains does not extend significantly outside of the subcortical telencephalon, demonstrating that white matter in the subcortical telencephalon of the constitutive Phr1 knockout is due to local neurons rather than inputs from the thalamus or cortex (Supplementary Fig. S4). From these results we conclude that descending corticofugal axons in Phr1 mutants fail to exit the cerebral cortex by E18.5, and that thalamocortical axons fail to reach their targets in the cortex by E18.5.

The defects in thalamic projections in the Phr1 mutant may reflect pathfinding errors of axons negotiating specific guidance choice points. Axons from the dorsal thalamus in the Phr1 mutant extend beyond their nuclei, but avoid the telencephalon, and instead, extend aberrantly along the diencephalon-telencephalon boundary, while wild-type thalamic axons cross this boundary on their way to the cortex (Fig. 6E,F). Mice deficient for the transcription factor Mash-1 exhibit a defect of thalamic axon outgrowth that is very similar to that of the Phr1 knockout (Tuttle et al. 1999). The Mash-1 defect may be due to the improper differentiation or migration of cells constituting a permissive corridor of Islet1-positive cells 

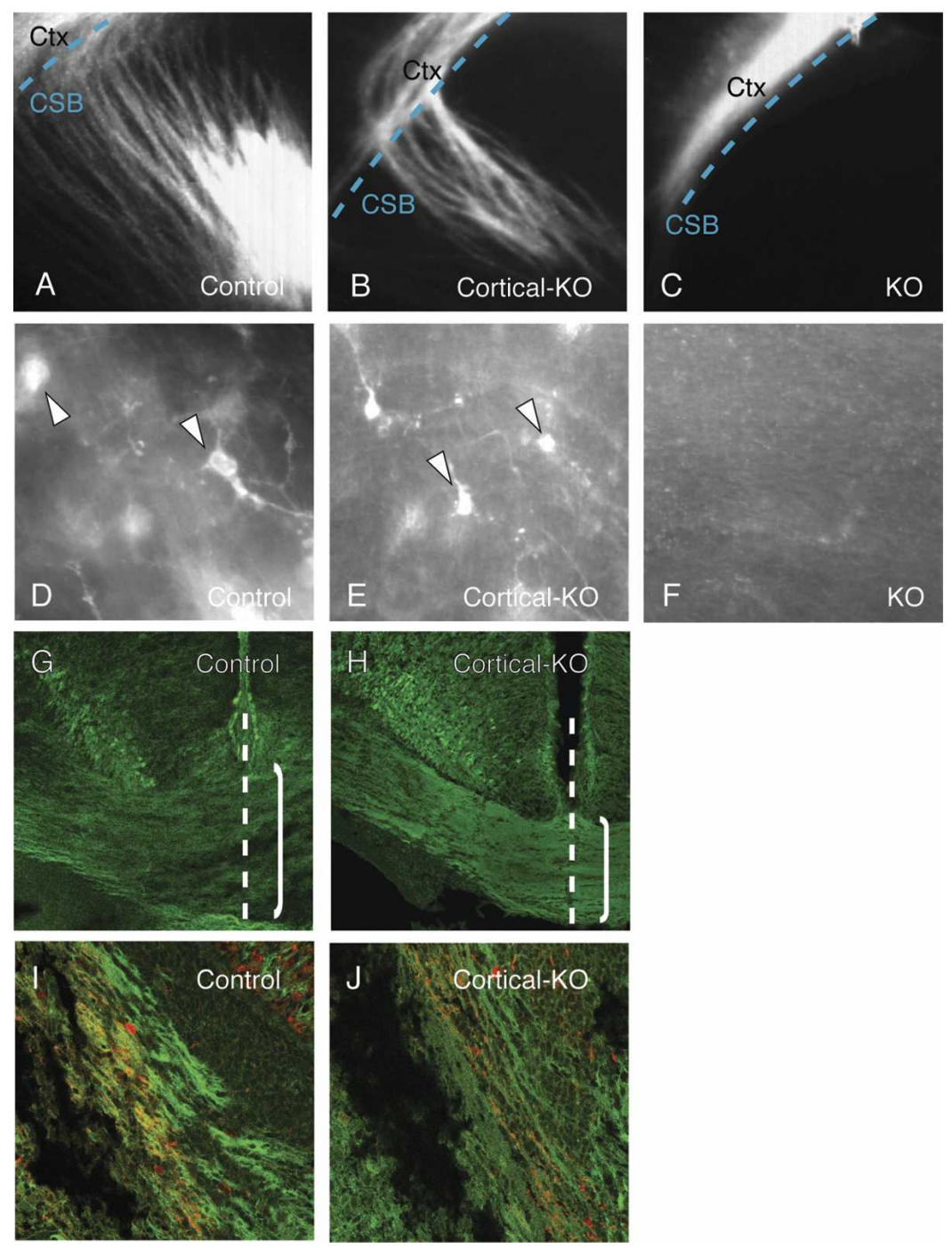

Figure 5. The cortical axon outgrowth defect of Phr1 mutants is cell-nonautonomous. DiI labeling is shown in E18.5 coronal sections in $A-F$. DiI placed in the somatosensory cortex $(\mathrm{Ctx})$ of control $(A)$ and cerebral cortex-specific Phr1 knockouts (Cortical-KO) (B) labels axons that cross the corticostriatal boundary $(\mathrm{CSB}$, dashed line) and traverse the internal capsule. $(C)$ When DiI is placed in the somatosensory cortex of the constitutive Phr1 knockouts (KO), axons remain in the cortex. When DiI is placed in the visual cortex of control $(D)$ or the cerebral cortex-specific Phr1 knockout $(E)$, cell bodies in the dorsal thalamus are labeled, demonstrating that thalamocortical projections have reached the cortex in these genotypes. $(F)$ DiI placement in the visual cortex of constitutive Phr1 knockouts (KO) fails to label retrogradely thalamic neurons. To visualize selectively Cre-expressing cortical neurons, Thy1-stop-YFP transgene mice were mated to produce control (Emx1-Cre, Thy1-stop-YFP) $(G, I)$ and the cerebral cortex-specific Phr1 knockouts (Cortical-KO: Emx1-Cre, Phr1 $1^{\text {flox }} / P h r 1^{\text {flox }}$, Thy1stop-YFP $(H, J)$. YFP-positive axons cross the midline (dashed line) and contribute to the corpus callosum (bracketed region) in both the control $(G)$ and cortical knockout $(H)$. Note that the corpus callosum is thinner in the cortical knockout as expected for a Phr1 knockout. YFP-positive axons also contribute to the internal capsule in both the control $(I)$ and cortical knockout $(J)$. In $I$ and $J$, Cre-expressing cortical axons in the internal capsule are labeled with YFP (green), while thalamocortical axons in the internal capsule are labeled with calretinin (red). The presence of cortical axons in the internal capsule of the cerebral cortex-specific Phr1 knockout, but not the constitutive Phr1 knockout, demonstrates that this cortical axon defect is non-cellautonomous. traversing the subcortical telencephalon (Lopez-Bendito et al. 2006). However, a well-formed domain of Islet1positive cells is frequently present in the subcortical telencephalon of Phr1 knockouts at E13.5, as it is in controls (Supplementary Fig. S5), demonstrating that the thalamic axon guidance defects cannot be due to loss of this corridor.

\section{Phrl regulates cortical axon outgrowth cell-nonautonomously}

Defects of cortical axon guidance through the corpus callosum are indistinguishable between the constitutive and cortex-specific knockouts; however, cortical axon guidance through the internal capsule is strikingly different for these two genotypes. Unlike the constitutive knockout, cerebral cortex-specific knockouts possess an internal capsule apparent by Nissl staining and containing axons positive for Neurofilament, L1, and calretinin (data not shown). Experiments placing DiI in the incipient somatosensory (Fig. 5B) or visual cortices (data not shown) at E18.5 also show labeled tracks running through the internal capsule and retrogradely labeled cell bodies in the thalamus (Fig. 5B,E).

To investigate this difference in the projection of descending cortical axons between the constitutive and cortex-specific knockouts, we tested in the cortex-specific knockout whether (1) descending corticofugal projections contribute to the internal capsule and (2) whether such cortical axons express Cre. The Emx1-Cre line is reported to express Cre in all projection neurons of the cortex (Gorski et al. 2002). To visualize selectively these Cre-expressing neurons, we used a Thy1-stop-YFP transgene that expresses YFP in neurons only if Cre excises a stop cassette that otherwise blocks expression. The transgene was crossed into both the control and the cortex-specific Phr1 knockout. Consistent with the results described above, Cre-expressing, cortically derived Phr1 knockout axons contribute to a thinner than normal corpus callosum in the cortex-specific knockout (Fig. 5G,H). In addition, Cre-expressing cortical neurons form corticofugal projections in the internal capsule of 
Figure 6. Axonal projections in the Phr1 mutant reveal guidance cues at the corticostriatal boundary. (A) DiI placed in the somatosensory cortex (Ctx) of controls at E14.5 labels axons that cross the corticostriatal boundary (CSB, dashed line). The boxed region is magnified in $A^{\prime}$ and highlights axons (arrowheads) extending deep into the subcortical telencephalon. $(B)$ DiI placed in the somatosensory cortex $(\mathrm{Ctx})$ of constitutive Phr1 knockouts (KO) at E14.5 labels axons that stop at the corticostriatal boundary (CSB, dashed line). The boxed region is magnified in $B^{\prime}$ and reveals the absence of axons extending into the subcortical telencephalon. $(C)$ At E15.5, DiI placed in the somatosensory cortex (Ctx) of controls labels axons that have crossed the diencephalon-telencephalon boundary (DTB, dashed line) and entered the thalamus (Th). (D) At E15.5, DiI placed in the somatosensory cortex (Ctx) of constitutive Phr1 knockouts (KO) labels axons that remain stalled at the corticostriatal boundary. These results demonstrate that cortical axons in the Phr1 mutant do not cross the corticostriatal boundary. (E) DiI placed in the thalamus (Th) of controls at E14.5 labels thalamic axons that cross the diencephalon-telencephalon boundary (DTB, dashed line) and enter the telencephalon. (F) DiI placed in the thalamus (Th) of constitutive Phr1 knockouts (KO) at E15.5 labels thalamic axons that fail to cross the diencephalon-telencephalon boundary (DTB, dashed line), but instead course ventrally along the boundary between telencephalon and hypothalamus. $(G)$ A schematic summarizes the results from control, cerebral cortex-specific Phr1 knockouts (Cortical-KO), and constitutive

Phr1 knockouts (KO) presented in Figures 5 and 6, where wild-type tissue is green and Phr1 mutant tissue is pink. Labeled structures include the cerebral cortex (Ctx), thalamus (Th), hypothalamus (Hyp), corpus callosum (CC), internal capsule (IC), corticostriatal boundary (CSB), and diencephalon-telencephalon boundary (DTB). The schematic highlights that, in the constitutive Phr1 knockout, (1) the cortical defect is non-cell-autonomous and (2) the corticofugal and thalamocortical axons fail to cross the corticostriatal boundary (CSB) and diencephalon-telencephalon boundary (DTB), respectively, and thus both avoid the subcortical telencephalon.

both controls and cortex-specific Phr1 knockouts (Fig. $5 \mathrm{I}, \mathrm{J})$, in contrast to cortical neurons in the constitutive Phr1 knockout, which do not contribute to an internal capsule. As a second method to assess whether Emx1Cre efficiently excises the floxed Phr 1 allele throughout the cerebral cortex and hippocampus, we examined wildtype and mutant Phr1 mRNA in the relevant brain regions of Emx1-Cre, floxed-Phr1 mice, and controls. RTPCR from E16.5 cortex demonstrates that wild-type Phr1 transcripts are undetectable in the presence of Emx1-Cre (Supplementary Fig. S6). Furthermore, since GABAergic interneurons in the cortex do not express Emx1-Cre (Gorski et al. 2002), these RT-PCR results suggest that GABAergic interneurons do not express appreciable amounts of Phr1 message, and are thus unlikely to contribute directly to the callosal or internal capsule defects caused by loss of Phr1. In combination, our results demonstrate that loss of cortical axons contributing to the internal capsule in Phr1 constitutive mutants is a cellnonautonomous defect most likely resulting from loss of Phr1 in cell bodies outside of the cerebral cortex.
The Phrl phenotype reveals a choice point for cortical axons at the corticostriatal boundary

Further analysis of the Phr1 constitutive mutants revealed that descending axons extend through the cerebral white matter and terminate abruptly at the border between the cortex and the striatum (Fig. 5C). The absence of cortical axons in the subcortical telencephalon is due to the failure of axons to cross the corticostriatal boundary rather than a retraction of axons to this boundary. At E13, cortical axons from both wild-type and constitutive Phr1 knockout animals extend within the cortex (data not shown). By E14.5, wild-type cortical axons have crossed the corticostriatal boundary (Fig. 6A) and extend far into the incipient striatum (Fig. 6A'). In contrast, at E14.5, cortical axons from constitutive Phr1 mutants extend through the cerebral white matter but terminate at the edge of the cortex in a bulb reminiscent of a Probst bundle (Fig. 6B, $\mathrm{B}^{\prime}$ ). By E15.5, the wild-type axons have reached the thalamus, while the Phr1 axons remain at the corticostriatal boundary (Fig. 6C,D). In 
normal development, thalamocortical and corticothalamic axons contact their reciprocal projections in the subcortical telencephalon and are subsequently guided along one another to their target tissues (Hevner et al. 2002). The cortical axon defect in Phr1 mutants, however, cannot be secondary to lack of direct contact with thalamic axons since cortical axons in Phr1 mutants never exit the cortex (Fig. 6). In contrast, in mutants with cortical projection defects secondary to thalamic projection defects, such as the Gbx2 knockout (Hevner et al. 2002), cortical axons cross the corticostriatal boundary and stall within the subcortical telencephalon.

Since descending corticofugal axons usually exit the cortex without contacting thalamic axons, the absence of such crossing in the Phr1 constitutive knockout cannot be due to the absence of direct contact with thalamic axons. However, the presence of the descending corticofugal projection in the cerebral cortex-specific Phr1 mutant could still be promoted by the eventual arrival of thalamic axons at the border of the corticostriatal boundary. If this were the case, then one would expect the cortical knockouts to display an identical cortical axon outgrowth defect to that of the complete Phr1 knockout at early stages, but later recover normal outgrowth once thalamic axons arrive. This was not observed. Experiments performed placing DiI in either the cortex or thalamus show results for the cortex-specific knockout similar to those of controls at all time points: At E12.5, axons do not extend out of the cortex of experimental or control animals (data not shown), but at E13.5, before thalamic axons reach the cortex, cortical axons have begun to turn the corner into the subcortical telencephalon from both control and experimental animals (Supplementary Fig. S7), and by E15.5, cortical axons in both genotypes have entered the thalamus (Supplementary Fig. S7). Therefore the cell-nonautonomous outgrowth defect of Phr1 mutant cortical axons is not rescued by the presence of wild-type thalamic axons in the internal capsule. In aggregate, our results show that the failure of cortical axons to cross the corticostriatal boundary in the constitutive Phr1 knockout is not due to the absence of Phr1 from either cerebral cortical neurons or glia of the Emx1-expressing lineage or from thalamic neurons that normally extend axons to the cortex. Instead, our results suggest that $P h r 1$ is required in a population of neurons or glia in the subcortical telencephalon that permits cortical axons to cross the corticostriatal boundary. This highlights a previously undemonstrated role for the corticostriatal boundary as a choice point for descending cortical axons.

\section{Phrl does not regulate CNS axon tract formation by down-regulating $D L K$}

In flies and worms, the ubiquitin ligases Highwire/ Rpm-1 down-regulate levels of the MAPKKKs Wallenda/ DLK, and the major morphological defects associated with highwire/rpm-1 loss of function are due to excess Wallenda/DLK. The strongest evidence for this conclusion is that the highwire/rpm-1 morphology phenotypes are suppressed in double mutants lacking both highwire/ rpm-1 and wallenda/DLK. To perform parallel experiments in mice, DLK mutants were generated (Supplementary Fig. S8A). The DLK knockout lacks an anterior commissure (Supplementary Fig. S8B,C), but the internal capsule is readily apparent in the mutant (Supplementary Fig. S8D,E). This phenotype is very similar to that recently described for an independently generated allele of $D L K$ (Hirai et al. 2006). To investigate whether the Phr1 CNS phenotype is due to excess DLK, Phr1 and DLK mutants were mated to produce double knockouts. These animals exhibit the same gross defects of brain morphology observed in the Phr1 single mutant, includ-
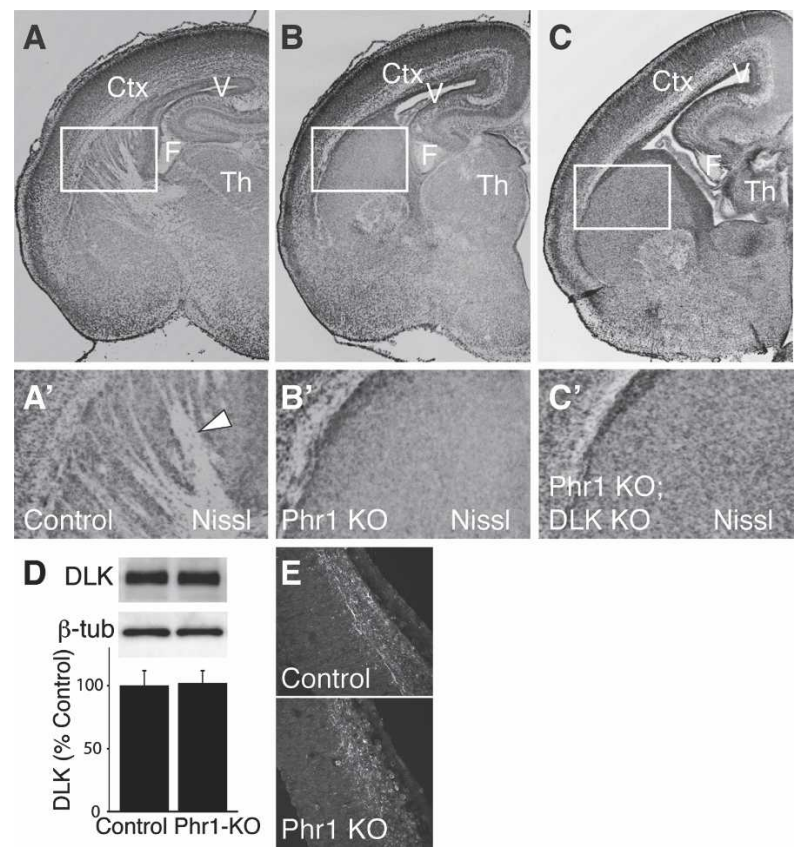

Figure 7. CNS defects persist in Phr1, DLK double mutants. Nissl-stained coronal sections from one side of E18.5 brains of control $\left(A, A^{\prime}\right), P h r 1$ constitutive knockout $(P h r 1 \mathrm{KO})\left(B, B^{\prime}\right.$, and Phr1; DLK double knockouts (Phr1 KO; DLK KO) $\left(C, C^{\prime}\right)$. In the Phr1 knockout and Phr1, DLK double knockout, lateral ventricles are increased in size and axon tracts running through the internal capsule are lacking. Cortex $(\mathrm{Ctx})$, lateral ventricle $(\mathrm{V})$, and fimbria $(\mathrm{F})$ are labeled. The boxed regions shown in $A^{\prime}, B^{\prime}$, and $C^{\prime}$ demonstrate the presence of white matter tracts (arrowhead) in the internal capsule from the control $\left(A^{\prime}\right)$ but not from the Phr1 knockout $\left(B^{\prime}\right)$ or Phr1, DLK double knockout $\left(C^{\prime}\right)$. These data demonstrate that the major CNS defects in the Phr1 knockout are not suppressed by mutating $D L K$. In $D$, Western analysis is shown for DLK and $\beta$-tubulin ( $\beta$-tub) from heads of conditional Phr1 knockout (Phr1-KO) and matching controls. Histogram shows DLK levels relative to $\beta$-tubulin loading controls and normalized to control levels. There are no significant differences in the level of DLK between the constitutive Phr1 knockout and control ( $n=4$ for each genotype). ( $E$ ) Immunohistochemical localization of DLK in cortical sections of E13.5 cerebral cortex from a constitutive Phr1 knockout (Phr1 KO) and matching control demonstrates no apparent difference in levels or localization of DLK protein. Data are presented as mean \pm SEM. 
ing the absence of an internal capsule (Fig. 7A-C). We also find no evidence for suppression of the synaptic sprouting defect at the NMJ of Phr1, DLK double mutants (data not shown), although the $D L K$ single mutant has occasional synaptic sprouts, making it more difficult to assess suppression.

To test whether Phr1 regulates the levels of DLK as demonstrated for the invertebrate orthologs, we measured protein levels of DLK by Western analysis from the heads of Phr1 constitutive knockouts. We observe no difference in the levels of DLK at time points relevant to the development of major axon tracts (Fig. 7D). In addition, we observe no detectable difference in the levels or localization of DLK protein in the embryonic cortex of Phr1 mutant brains as assessed by immunohistochemistry (Fig. 7E). Levels of DLK from heads of Phr1 deficiency embryos are also not greater than in controls $186 \pm 6 \%$ of control, $n=3, P>0.2$ ). Finally, we measured the levels of LZK, the closest mammalian homolog of DLK (Sakuma et al. 1997), and found by Western analysis of embryonic heads no significant difference between wild-type and the Phr1 knockout $(110 \pm 14 \%$ of control, $n=4, P>0.6)$. Hence, we have no evidence for increased levels of DLK or LZK in the brain of Phr1 mutants. These data, in combination with the double-mutant analysis above, demonstrate that the Phr1 CNS mutant phenotype is not due to an inability to down-regulate DLK.

\section{Discussion}

An evolutionarily conserved role for Phrl in neuromuscular development

Invertebrate orthologs of Phr1 are required for normal neuromuscular development. Here we demonstrate evolutionary conservation of this function for Phr1 in mammals. In both Drosophila and mouse, the fine structure of NMJ terminal morphology is easily assessed and demonstrates both similarities and differences between phenotypes of highwire flies and Phr1 mice. In the fly, absence of highwire leads to excessive branching of the motoneuron axon and a large increase in the number of synaptic boutons on the muscle (Wan et al. 2000). In the mouse, motoneuron terminals also show extra branching with a profusion of extrasynaptic sprouts (Fig. 1F,G; Burgess et al. 2004). In both fly and mouse these phenotypes reflect a cell-autonomous requirement for Phr1/ highwire in the motoneuron and suggest a role in restraining nerve terminal branching. However, unlike in fly, the excess sprouts in the mouse do not form synapses, likely reflecting differences in the mechanism of NMJ formation in flies and mice. At the fly NMJ, each muscle cell is confronted by a branched axon forming arbors of synaptic boutons, so the additional branches in a highwire mutant may utilize the normal muscle machinery for building such boutons. In the mouse, in contrast, each muscle fiber has only a single endplate (Sanes and Lichtman 1999|, and may not be receptive to building ectopic post-synaptic structures in response to additional presynaptic branches.
Phrl is required for the formation of major CNS axonal projections

The thin phrenic nerve and partial innervation of the diaphragm in the Phr1 mutant indicate functions for Phr1 beyond the regulation of nerve terminal morphology, suggesting defects of motor axon guidance to their muscle targets. This is in contrast to Drosophila highwire mutants, which display normal motoneuron axon guidance and complete innervation of all target muscles (Wan et al. 2000). Retinal ganglion cell axons display more dramatic guidance defects, growing out of the retina, but stalling within the optic nerve or at the optic chiasm. Indeed, there is a severe and specific loss of axon tracts throughout the CNS: The corpus callosum is reduced in thickness due to a loss of axons crossing from the posterior cerebral cortex, the anterior commissure is entirely lacking, and thalamic and cortical axons fail to enter the subcortical telencephalon to form the internal capsule. While Phr1 mutant axons could have general defects in axon growth, the phenotypes are more consistent with defects in axon guidance. Axons stall at specific boundaries including the diencephalon-telencephalon border and the corticostriatal boundary, and axons originating from the same region of the cerebral cortex fail to enter the striatum but successfully cross the corpus callosum. Hence, Phr1 not only regulates the fine structure of synaptic connections like its invertebrate cousins, but also is required for the large-scale pattern of neuronal connectivity in the mammalian brain.

\section{Phr1 regulates axon guidance in the CNS cell-nonautonomously}

All previously described loss-of-function phenotypes for orthologs of Phr1 are cell-autonomous (Schaefer et al. 2000; Zhen et al. 2000; D'Souza et al. 2005; Wu et al. 2005). Therefore, axon guidance defects in Phr1 mutants would naturally be assumed due to a misreading of molecular guidance cues by Phr1 mutant axons, and this may be the case for callosal cortical axons. However, for descending cortical axons, this is not the case. When Phr1 function is deleted from neurons in the cerebral cortex using Emx1-Cre, the internal capsule forms, cortical neurons innervate the thalamus and vice versa, and Cre-positive marked cortical axons pass through the subcortical telencephalon (data summarized in Fig. 6G). As surprising as this result is, it is not unique to cortical axons. In the Phr1 constitutive knockout, retinal ganglion cell projections fail to reach their targets; however, when Phr1 is deleted selectively in the retina, mutant axons fully innervate their targets in the thalamus, revealing a non-cell-autonomous requirement for Phr1 outside of the retina for ganglion cell axon pathfinding (S. Cullican, A.J. Bloom, and A. DiAntonio, unpubl.).

Cell-nonautonomous defects of cortical axon guidance occur in mutants with severely defective thalamocortical tracts (Hevner et al. 2002), suggesting that cortical axons require thalamic axons to reach their targets in the thalamus. During normal development, cortical pioneer 
axons cross the corticostriatal junction and enter the internal capsule quickly at approximately E13.5, but having reached the middle of the internal capsule by E14.5, appear to pause in the presumptive neostriatum for 2448 h. Jacobs et al. (2007) speculate that this pause in the neostriatum indicates the time required for cortical axons to gather and "match up" corresponding thalamocortical efferents. Indeed, the cortical axon defects in mutants lacking thalamic axons are consistent with a missed appointment; i.e., cortical axons have extended midway into the subcortical telencephalon at E14.5 but progress no further (Hevner et al. 2002). This is not the case in Phr1 mutants. Rather, cortical axons in complete Phr1 mutants appear to avoid the incipient striatum, whereas cortex-specific Phr1 knockouts extend their axons into the internal capsule normally at all developmental time points. This is evidence that the cortical axon defect in Phr1 mutants is not secondary to loss of thalamic axons, but is instead akin to the specific pathfinding defect of the thalamic projection in Phr1 mutants.

The Phrl mutant reveals a choice point for cortical axons at the corticostriatal boundary

An axon's course is influenced by attributes of the projecting neuron and its final target, as well as by factors regulating the molecular environment along its path. This environment consists of permissive and nonpermissive substrates for growth, and diffusible factors that promote growth toward targets or repel axons away from improper targets (Tessier-Lavigne and Goodman 1996). Hence, the improper development and organization of cells intervening between a projecting neuron and its target lead to cell-nonautonomous defects in axonal outgrowth and guidance (Tuttle et al. 1999; Lopez-Bendito et al. 2006). Boundaries between differing tissues can be choice points for growing axons; there is strong evidence for this at the diencephalon-telencephalon boundary (Tuttle et al. 1999; Jacobs et al. 2007). In Phr1 knockouts, thalamocortical axons stop at this boundary. The corticostriatal boundary is an obvious anatomical landmark, a boundary between molecularly distinct cell types in the telencephalon and an identified boundary for thalamic axons entering the cortex (Molnar and Blakemore 1995; Chapouton et al. 2001; Inoue et al. 2001; Jones et al. 2002; Molnar and Butler 2002; Carney et al. 2006). However, previously identified mutants with cortical axon defects either extend their axons past this boundary into the striatum (Bagri et al. 2002; Hevner et al. 2002; Tissir et al. 2005) or display defects interpreted as affecting axon outgrowth within the cortex (Wang et al. 2002). In contrast, cortical axons in the Phr1 knockout proceed to the corticostriatal boundary and stop. These results reveal the corticostriatal boundary as a choice point for axons exiting the cortex. We show here that the failure of cortical axons to cross this boundary in the constitutive Phr1 knockout is not due to the absence of Phr1 in either cerebral cortical neurons or glia of the Emx1-expressing lineage or thalamic neurons that normally extend axons to the cortex. Instead, these results suggest the presence of an uncharacterized population of neurons or glia in the subcortical telencephalon that require $P h r 1$ to make this boundary passable to cortical axons. This inability of cortical axons to enter the subcortical telencephalon is very similar to the defect in mutant thalamic axons that also fail to enter the subcortical telencephalon, leading us to suspect a common cell-nonautonomous mechanism. These findings suggest a model in which Phr1 is required to render the subcortical forebrain permissive to the entry of both cortical and thalamic axons.

\section{Phr1 CNS axon tract phenotypes do not require DLK}

Large-scale unbiased genetic screens in both flies and worms have demonstrated that the MAPKKK Wallenda/ DLK is required for the major morphological phenotypes observed when highwire/rpm-1 is mutant (Nakata et al. 2005; Collins et al. 2006). In these systems Highwire/ Rpm-1 regulates the levels of Wallenda/DLK, and the up-regulation of Wallenda/DLK is both necessary and sufficient to cause synaptic morphology defects in highwire and rpm-1 mutants (Nakata et al. 2005; Collins et al. 2006). The striking evolutionary conservation between flies and worms lead us to suspect that this pathway would also be central to the Phr1 phenotype in mammals. However, we observe no change in the levels or localization of DLK or its close homolog LZK in the developing CNS of Phr1 mutant mice. Furthermore, mutations in $D L K$ do not suppress the gross morphological changes in the CNS-such as loss of the internal capsule- that are the hallmark phenotypes of the Phr1 mutant. These results do not show that $P h r 1$ never regulates DLK, but they do demonstrate that, unlike in invertebrates, up-regulation of DLK is not necessary for major morphological phenotypes of the Phr1 mutant, indicating mechanisms not anticipated by studies in invertebrates.

\section{Materials and methods}

\section{Phr1 and DLK mice}

A targeting vector was constructed from PCR products from embryonic stem (ES) cell genomic DNA from the 129 cell line subsequently used to generate the Phr1 targeted allele mice. Three PCR fragments, adjacent to one another in the genomic DNA, were amplified: a 1.3-kb "short arm," a 1.6-kb "floxed region" containing translated exons 8 and 9, and a 3-kb "long arm." The 3' primer used to generate the floxed region also included the 34-base-pair (bp) loxP sequence in order to introduce a loxP site between the floxed region and the long arm. Each fragment was subcloned into vector pGEM Easy (Promega) and multiple clones from each ligation were sequenced. Accurate copies of each fragment were cloned in order into vector pSV-LoxP-FRT-neo-FRT, which contains a TN5 Neo casette flanked by FRT sites adjacent to a second loxP site. Thus, exons 8 and 9 were flanked by loxP sites. The construct was electroporated into ES cells and homologous recombinants containing both loxP sites were confirmed by Southern blot. Two different clones were injected into mouse blastocysts to generate germ- 
line chimeras. Germline chimeras were bred to C57 Black mice to produce heterozygotes for the floxed allele. Germline chimeras were also bred to the $\beta$-actin-Cre line (Lewandoski and Martin 1997) to delete the floxed exons in the germline and produce the constitutive Phr1 knockout allele. In this knockout, RTPCR demonstrates that these exons are missing from the transcribed RNA, leaving a transcript that could encode a protein carrying an internal deletion. Constitutive alleles generated by the two clones were judged to have identical phenotypes and subsequently were treated identically.

The $D L K$ gene trap mutant chimeras were generated by injecting blastocysts with murine ES cells from line RRN366 (BayGenomics Consortium) containing the gene trap construct pGTOLxf inserted in an intron in the $5^{\prime}$ untranslated region of DLK (see http://baygenomics.ucsf.edu). Germline chimeras were bred to C57 Black mice to produce heterozygotes for the gene trap allele. Offspring were subsequently mated to mice with the targeted Phr1 knockout allele to ultimately generate DLK gene trap, Phr1 knockout double homozygotes.

\section{Mice}

The presence of different Phr1 alleles was determined by PCR on tail DNA using three different primers: a 5' primer from from the Neo cassette /CACACATTCCACAGCTGGTTCTTTCC GCCTCAG), a $5^{\prime}$ primer from the $P h r 1$ locus (TTTTTCAATC CCAAATAAAGGTGTTAGAAG), and a 3 ' primer from the Phr1 locus (ACACAGACGACGTCTGCAGCTTCAAGAGAA).

$15 \mathrm{DttMb}$ and $1 \mathrm{Acrg}$ piebald deletion stock mice (Roix et al. 2001) were obtained from Timothy P. O'Brien (Cornell University, Ithaca, NY). Embryos and neonatal mice referred to in this text as "deficiency" mice were obtained by mating together $15 \mathrm{DttMb}$ and $1 \mathrm{Acrg}$ stock mice or $15 \mathrm{DttMb}$ mice with one another. Thy-Stop-YFP15 (Feng et al. 2000), Emx-1 Cre (Gorski et al. 2002), HB9-Cre (Fox et al. 2007), and $\beta$ actin-Cre mice (Lewandoski and Martin 1997) were as describe previously.

All embryos were collected by Caesarian section from timed matings where detection of the vaginal plug was defined as E0.5. For cell proliferation studies, pregnant mice were injected with $50 \mathrm{mg} / \mathrm{kg} \mathrm{BrdU}$ (Roche) at E12.5 or E15.5.

\section{Southern blot}

Genomic DNA prepared from ES cell cultures or mouse liver was cut with ApaI and PacI. Radioactive probes were generated using the Rediprime II Random Prime Labeling System (Amersham) from one of two PCR fragments: $5^{\prime}$ of the construct generated from primers GTATTTTTACTCAAGAATCATATAAA TTTGA and AAAGCTAAACGAAGACCTCATGTGC or $3^{\prime}$ of the construct generated from primers AGTAGGCTTTGAGTT TTTAAAAGCCCATCC and AAAATCTAAAGTGTCTTTTTA TATCTTCTC. Probes were passed over ProbeQuant G-50 Micro Columns (Amersham). Radiolabeling was detected with a PhosphorImaging plate (Amersham) and Molecular Imager FX (Bio-Rad)

\section{$R T-P C R$}

RNA was extracted from dissected (1) cortex and hippocampus or (2) the remaining brain using TRIzol reagent (Invitrogen) and RT-PCR was performed using the SuperScript One-Step RTPCR kit (Invitrogen). Primers were AAACAGATTATTGAG GTTGGGCTTTGTGAA and AAAAGGGCATGCGAGCCAT CATGTCCCACA, which flank the floxed domain. The reaction incubated at $55^{\circ} \mathrm{C}$ for $30 \mathrm{~min}$, followed by 40 cycles of 15 sec denaturing at $94^{\circ} \mathrm{C}, 30 \mathrm{sec}$ annealing at $60^{\circ} \mathrm{C}$, and $1 \mathrm{~min}$ extension at $68^{\circ} \mathrm{C}$.

\section{Histology}

For whole-mount nerve and muscle staining, tissue was fixed in $2 \%$ paraformaldehyde-PBS for $2 \mathrm{~h}$. Muscle was incubated in rabbit anti-neurofilament 200 (Sigma, 1:80) or mouse anti-panneurofilament cocktail (SMI, 1:1,000) and rabbit anti-synaptophysin (Zymed, 1:500) for at least $24 \mathrm{~h}$ at room temperature. Secondary antibodies used were $\mathrm{Cy} 3$ goat $\alpha$-rabbit and Alexa 488-conjugated Bungarotoxin (both from Molecular Probes, $1: 1000)$.

For immunohistochemical analysis, with the exception of $\alpha$-GABA, brains were fixed overnight at $4{ }^{\circ} \mathrm{C}$ in $4 \%$ paraformaldehyde-PBS, sunk in $30 \%$ sucrose, and frozen in OTC, cut into $20-\mu \mathrm{m}$ floating sections with a cryostat and mounted on Superfrost Plus glass slides. For staining with $\alpha$-GABA, brains were fixed $2 \mathrm{~h}$ at $4^{\circ} \mathrm{C}$ in $2 \%$ paraformaldehyde-PBS and $14-\mu \mathrm{m}$ sections were cut; slides were post-fixed in $1 \%$ paraformaldehydePBS for 1 min and incubated serialy with Fab fragment (1:100); $0.1 \mathrm{M}$ glycine in PBS, $0.5 \%$ sodium borohydride; and finally $3 \%$ normal goat serum, $1 \%$ Triton X-100 in PBS before rabbit $\alpha$-GABA (Sigma, 1:500). Other primary antibodies were rat $\alpha$-L1 (Chemicon, 1:100), mouse $\alpha$-pan-neurofilament cocktail (SMI, $1: 1000$ ), rabbit $\alpha$-calretinin (Swant, 1:2000) rabbit $\alpha$-Islet (provided by T. Jessell, Columbia University, New York, 1:5000), rabbit $\alpha$-DLK C-terminal (Holzman et al. 1994, 1:500) (provided by L. Holzman, University of Michigan, Ann Arbor, MI), and rabbit $\alpha$-Tbr-1 (provided by R.F. Hevner, University of Washington, Seattle, WA, 1:2500). Nissl-stained slides were incubated in cresyl violet. Fluorescent secondaries were Cy3 goat $\alpha$-rat, Cy3 goat $\alpha$-rabbit, and Alexa488 goat $\alpha$-mouse and Neurotrace 640/ 660 Nissl stain (all from Molecular Probes).

Cell death was assessed with rabbit $\alpha$-Cleaved caspase-3 (Cell Signaling, 1:200), as well as the In Situ Cell Death Detection Kit, TMR red (Roche), following the kit procedure. Mouse $\alpha$-BrdU (Beckton-Dickson, 1:100) was used to detect BrdU for analysis of cell proliferation.

\section{Western blotting}

For analysis of DLK levels in Phr1 knockout mice, whole heads were taken from E12.5 and E14.5 embryos and frozen in liquid $\mathrm{N}_{2}$. For analysis of DLK levels in the DLK gene trap mice, brains were dissected out of P7 animals, bisected with a mid-sagittal cut, and frozen in liquid $\mathrm{N}_{2}$. Whole heads or brains were homogenized in an ice-cold buffer containing 10 parts suspension buffer (100 mM NaCl, $100 \mathrm{mM}$ Tris-Cl at pH 8, 1 mM EDTA), four parts $8 \mathrm{M}$ urea, and 1 part $20 \%$ SDS. Samples were then diluted in $2 \times$ SDS loading buffer ( $100 \mathrm{mM}$ Tris-Cl at pH 6.8, 200 mM DTT, 4\% SDS, $0.2 \%$ bromophenol blue, $20 \%$ glycerol), boiled for $10 \mathrm{~min}$, and passed through a 23-G needle. Proteins were separated using a $4 \%-20 \%$ SDS-PAGE gel (Cambrex) and transferred to PVDF membrane (Bio-Rad). Membranes were blocked in a solution containing $0.1 \%$ Tween 20 (Amresco) and $5 \%$ nonfat dry milk in PBS. Blots were then labeled with the following primary antibodies diluted in blocking buffer: DLK C-terminal (a gift from Lawrence B. Holzman, University of Michigan, Ann Arbor, MI, 1:500), or $\beta$-tubulin (Developmental Hybridoma Bank, 1:100). Blots were then labeled with the appropriate HRP-conjugated secondary antibody and developed using LumiLight Plus solution (Invitrogen).

\section{DiI labeling}

After DiI crystal placement, fixed brains were returned to $4 \%$ paraformaldehyde-PBS at room temperature. E12.5-E15.5 brains remained in fix at least $10 \mathrm{~d}, \mathrm{E} 18.5$ brains at least $14 \mathrm{~d}, \mathrm{P} 6$ and P8 at least $21 \mathrm{~d}$. One-hundred-micron to $200-\mu \mathrm{m}$ sections were cut on a vibratome. Corticostriatal boundaries were estimated 
by following the arc of the lateral ventricles and cerebral cortex ventrally; diencephalon-telencephalon boundaries in sections were evident from tissue morphology.

\section{Imaging and analysis}

Images of Nissl-stained sections and DiI-labeled sections were obtained with a Cool SNAP cf camera (Photometrics) mounted on an Eclipse 80i microscope (Nikon). Images taken from slides labeled with fluorescent secondaries were acquired on a Nikon $\mathrm{C} 1$ confocal microscope. Samples for each experiment were imaged using the same confocal gain settings. Composite images were pieced together using Adobe Photoshop CS2. For axon and endplate counting and endplate band width measurements, stacks of confocal images were merged together using EZ-C1 software (Nikon). MetaMorph software (Molecular Devices) was used to measure average endplate band width by drawing a polygon around the endplate band in a merged image, measuring the area, and dividing by the endplate band length. Equivalent regions of the diaphragm muscle were chosen to image for endplate counting and band width measurement using the major branchpoint of the phrenic nerve on the muscle as a landmark. Quantification was performed while blinded to genotypes. BdrU-labeled cells were counted automatically using MetaMorph software (Molecular Devices) within blinded user-defined cortical domains. Images of Western blots were acquired using a ChemiDoc XRS scanner (Bio-Rad) and band intensities were measured using Quantity One software (Bio-Rad). The corpus callosum dorsal-ventral thickness and anterior-posterior length were measured from E18.5 brains cut into coronal sections by vibratome or cryostat. The cross-sectional area at the midline was estimated as the product of the measured length and thickness. Statistical analyses were performed and graphs were generated with Origin 7.0 (Origin Laboratory). Data sets were compared using the $t$-test or ANOVA where appropriate.

\section{Acknowledgments}

We are very grateful to Dr. Janice Brunstrom, Dr. Susan Culican, Dr. Steven Mennerick, Dr. David Ornitz, Dr. Joshua Weiner, Dr. Thomas Baranski, and Dr. Brad Miller for their advice and assistance. We also recognize the other members of the DiAntonio laboratory, especially Dr. Catherine Collins and Dr. Chunlai $\mathrm{Wu}$, and our skilled technician Sylvia Johnson. Electroporation of ES cells was preformed by the gracious staff of the Embryonic Stem Cell Core at the Siteman Cancer Center, and mouse colony maintenance was managed with care by the technicians of the Washington University Animal Facility. This work was supported by the Keck and McKnight Scholars Awards to A.D.

\section{References}

Bagri, A., Marin, O., Plump, A.S., Mak, J., Pleasure, S.J., Rubenstein, J.L., and Tessier-Lavigne, M. 2002. Slit proteins prevent midline crossing and determine the dorsoventral position of major axonal pathways in the mammalian forebrain. Neuron 33: 233-248.

Bareyre, F.M., Kerschensteiner, M., Misgeld, T., and Sanes, J.R. 2005. Transgenic labeling of the corticospinal tract for monitoring axonal responses to spinal cord injury. Nat. Med. 11: 1355-1360.

Burgess, R.W., Peterson, K.A., Johnson, M.J., Roix, J.J., Welsh, I.C., and O'Brien, T.P. 2004. Evidence for a conserved function in synapse formation reveals Phrll as a candidate gene for respiratory failure in newborn mice. Mol. Cell. Biol. 24:
1096-1105.

Carney, R.S., Alfonso, T.B., Cohen, D., Dai, H., Nery, S., Stocia, B., Slotkin, J., Bregman, B.S., Fishell, G., and Corbin, J.G. 2006. Cell migration along the lateral cortical stream to the developing basal telencephalic limbic system. I. Neurosci. 26: 11562-11574.

Chapouton, P., Schuurmans, C., Guillemot, F., and Gotz, M. 2001. The transcription factor neurogenin 2 restricts cell migration from the cortex to the striatum. Development 128: 5149-5159.

Collins, C.A., Wairkar, Y.P., Johnson, S.L., and DiAntonio, A. 2006. Highwire restrains synaptic growth by attenuating a MAP kinase signal. Neuron 51: 57-69.

DiAntonio, A., Haghighi, A.P., Portman, S.L., Lee, J.D., Amaranto, A.M., and Goodman, C.S. 2001. Ubiquitination-dependent mechanisms regulate synaptic growth and function. Nature 412: 449-452.

D'Souza, J., Hendricks, M., Le Guyader, S., Subburaju, S., Grunewald, B., Scholich, K., and Jesuthasan, S. 2005. Formation of the retinotectal projection requires Esrom, an ortho$\log$ of PAM (protein associated with Myc). Development 132: 247-256.

Englund, C., Fink, A., Lau, C., Pham, D., Daza, R.A., Bulfone, A., Kowalczyk, T., and Hevner, R.F. 2005. Pax6, Tbr2, and Tbr1 are expressed sequentially by radial glia, intermediate progenitor cells, and postmitotic neurons in developing neocortex. J. Neurosci. 25: 247-251.

Feng, G., Mellor, R.H., Bernstein, M., Keller-Peck, C., Nguyen, Q.T., Wallace, M., Nerbonne, J.M., Lichtman, J.W., and Sanes, J.R. 2000. Imaging neuronal subsets in transgenic mice expressing multiple spectral variants of GFP. Neuron 28: 41-51.

Fox, M.A., Sanes, J.R., Borza, D.B., Eswarakumar, V.P., Fassler, R., Hudson, B.G., John, S.W., Ninomiya, Y., Pedchenko, V., Pfaff, S.L., et al. 2007. Distinct target-derived signals organize formation, maturation, and maintenance of motor nerve terminals. Cell 129: 179-193.

Gorski, J.A., Talley, T., Qiu, M., Puelles, L., Rubenstein, J.L., and Jones, K.R. 2002. Cortical excitatory neurons and glia, but not GABAergic neurons, are produced in the Emx1-expressing lineage. J. Neurosci. 22: 6309-6314.

Guo, Q., Xie, J., Dang, C.V., Liu, E.T., and Bishop, J.M. 1998. Identification of a large Myc-binding protein that contains RCC1-like repeats. Proc. Natl. Acad. Sci. 95: 9172-9177.

Hevner, R.F., Miyashita-Lin, E., and Rubenstein, J.L. 2002. Cortical and thalamic axon pathfinding defects in Tbr1, Gbx2, and Pax6 mutant mice: Evidence that cortical and thalamic axons interact and guide each other. J. Comp. Neurol. 447: $8-17$.

Hirai, S., Cui, de F., Miyata, T., Ogawa, M., Kiyonari, H., Suda, Y., Aizawa, S., Banba, Y., and Ohno, S. 2006. The c-Jun Nterminal kinase activator dual leucine zipper kinase regulates axon growth and neuronal migration in the developing cerebral cortex. J. Neurosci. 26: 11992-12002.

Holzman, L.B., Merritt, S.E., and Fan, G. 1994. Identification, molecular cloning, and characterization of dual leucine zipper bearing kinase. A novel serine/threonine protein kinase that defines a second subfamily of mixed lineage kinases. $J$. Biol. Chem. 269: 30808-30817.

Inoue, T., Tanaka, T., Takeichi, M., Chisaka, O., Nakamura, S., and Osumi, N. 2001. Role of cadherins in maintaining the compartment boundary between the cortex and striatum during development. Development 128: 561-569.

Jacobs, E.C., Campagnoni, C., Kampf, K., Reyes, S.D., Kalra, V., Handley, V., Xie, Y.Y., Hong-Hu, Y., Spreur, V., Fisher, R.S., et al. 2007. Visualization of corticofugal projections during 
early cortical development in a $\tau$-GFP-transgenic mouse. Eur. I. Neurosci. 25: 17-30.

Jones, L., Lopez-Bendito, G., Gruss, P., Stoykova, A., and Molnar, Z. 2002. Pax6 is required for the normal development of the forebrain axonal connections. Development 129: 50415052 .

Lewandoski, M. and Martin, G.R. 1997. Cre-mediated chromosome loss in mice. Nat. Genet. 17: 223-225.

Lopez-Bendito, G., Cautinat, A., Sanchez, J.A., Bielle, F., Flames, N., Garratt, A.N., Talmage, D.A., Role, L.W., Charnay, P., Marin, O., et al. 2006. Tangential neuronal migration controls axon guidance: A role for neuregulin-1 in thalamocortical axon navigation. Cell 125: 127-142.

McCabe, B.D., Hom, S., Aberle, H., Fetter, R.D., Marques, G., Haerry, T.E., Wan, H., O'Connor, M.B., Goodman, C.S., and Haghighi, A.P. 2004. Highwire regulates presynaptic BMP signaling essential for synaptic growth. Neuron 41: 891-905.

Molnar, Z. and Blakemore, C. 1995. How do thalamic axons find their way to the cortex? Trends Neurosci. 18: 389-397.

Molnar, Z. and Butler, A.B. 2002. The corticostriatal junction: A crucial region for forebrain development and evolution. Bioessays 24: 530-541.

Murthy, V., Han, S., Beauchamp, R.L., Smith, N., Haddad, L.A., Ito, N., and Ramesh, V. 2004. Pam and its ortholog highwire interact with and may negatively regulate the TSC1.TSC2 complex. J. Biol. Chem. 279: 1351-1358.

Nakata, K., Abrams, B., Grill, B., Goncharov, A., Huang, X., Chisholm, A.D., and Jin, Y. 2005. Regulation of a DLK-1 and p38 MAP kinase pathway by the ubiquitin ligase RPM-1 is required for presynaptic development. Cell 120: 407-420.

Pierre, S.C., Hausler, J., Birod, K., Geisslinger, G., and Scholich, K. 2004. PAM mediates sustained inhibition of cAMP signaling by sphingosine-1-phosphate. EMBO J. 23: 3031-3040.

Reddy, U.R. and Pleasure, D. 1994. Cloning of a novel putative protein kinase having a leucine zipper domain from human brain. Biochem. Biophys. Res. Commun. 202: 613-620.

Roix, J.J., Hagge-Greenberg, A., Bissonnette, D.M., Rodick, S., Russell, L.B., and O'Brien, T.P. 2001. Molecular and functional mapping of the piebald deletion complex on mouse chromosome 14. Genetics 157: 803-815.

Sakuma, H., Ikeda, A., Oka, S., Kozutsumi, Y., Zanetta, J.P., and Kawasaki, T. 1997. Molecular cloning and functional expression of a cDNA encoding a new member of mixed lineage protein kinase from human brain. J. Biol. Chem. 272: 28622 28629.

Sanes, J.R. and Lichtman, J.W. 1999. Development of the vertebrate neuromuscular junction. Annu. Rev. Neurosci. 22: 389-442.

Santos, T.M., Han, S., Bowser, M., Sazani, K., Beauchamp, R.L., Murthy, V., Bhide, P.G., and Ramesh, V. 2006. Alternative splicing in protein associated with Myc (Pam) influences its binding to c-Myc. J. Neurosci. Res. 83: 222-232.

Schaefer, A.M., Hadwiger, G.D., and Nonet, M.L. 2000. rpm-1, a conserved neuronal gene that regulates targeting and synaptogenesis in C. elegans. Neuron 26: 345-356.

Tessier-Lavigne, M. and Goodman, C.S. 1996. The molecular biology of axon guidance. Science 2741: 123-133.

Tissir, F., Bar, I., Jossin, Y., De Backer, O., and Goffinet, A.M. 2005. Protocadherin Celsr3 is crucial in axonal tract development. Nat. Neurosci. 8: 451-457.

Tuttle, R., Nakagawa, Y., Johnson, J.E., and O'Leary, D.D. 1999. Defects in thalamocortical axon pathfinding correlate with altered cell domains in Mash-1-deficient mice. Development 126: 1903-1916.

Wan, H.I., DiAntonio, A., Fetter, R.D., Bergstrom, K., Strauss, R., and Goodman, C.S. 2000. Highwire regulates synaptic growth in Drosophila. Neuron 26: 313-329.

Wang, Y., Thekdi, N., Smallwood, P.M., Macke, J.P., and Nathans, J. 2002. Frizzled-3 is required for the development of major fiber tracts in the rostral CNS. J. Neurosci. 22: 8563-8573.

Wu, C., Wairkar, Y.P., Collins, C.A., and DiAntonio, A. 2005. Highwire function at the Drosophila neuromuscular junction: Spatial, structural, and temporal requirements. J. Neurosci. 25: 9557-9566.

Yang, H., Scholich, K., Poser, S., Storm, D.R., Patel, T.B., and Goldowitz, D. 2002. Developmental expression of PAM (protein associated with MYC) in the rodent brain. Brain Res. Dev. Brain Res. 136: 35-42.

Zhen, M., Huang, X., Bamber, B., and Jin, Y. 2000. Regulation of presynaptic terminal organization by C. elegans RPM-1, a putative guanine nucleotide exchanger with a RING-H2 finger domain. Neuron 26: 331-343. 


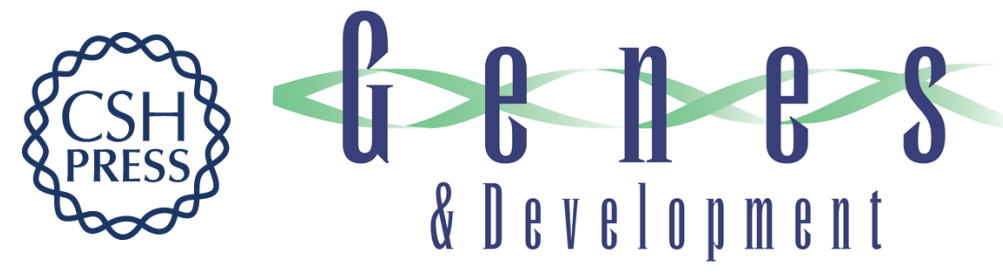

\section{The requirement for Phr1 in CNS axon tract formation reveals the corticostriatal boundary as a choice point for cortical axons}

A. Joseph Bloom, Bradley R. Miller, Joshua R. Sanes, et al.

Genes Dev. 2007, 21: originally published online September 27, 2007

Access the most recent version at doi:10.1101/gad.1592107

\section{Supplemental http://genesdev.cshlp.org/content/suppl/2007/09/28/gad.1592107.DC1 \\ Material}

References This article cites 41 articles, 16 of which can be accessed free at:

http://genesdev.cshlp.org/content/21/20/2593.full.html\#ref-list-1

License

Email Alerting

Receive free email alerts when new articles cite this article - sign up in the box at the top

Service

right corner of the article or click here.

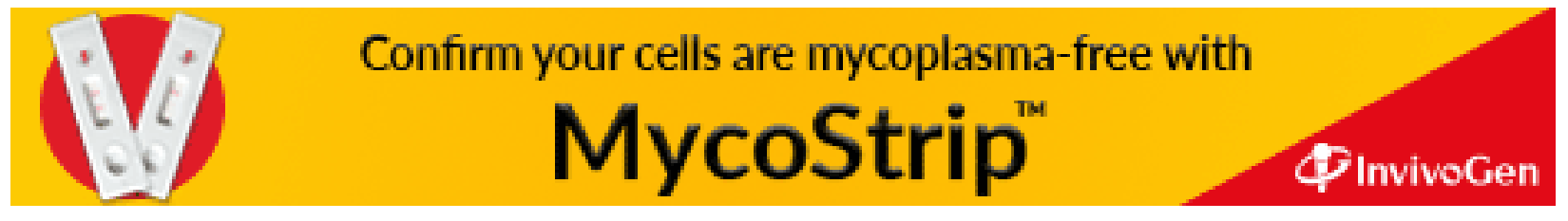

